\title{
Evaluation of Cardiovascular Risk in Hidradenitis Suppurativa Patients Using Heart Rate Variability (HRV) Analysis
}

\author{
Nevena Skroza, ${ }^{1}$ Alessandra Mambrin, ${ }^{1}$ Ilaria Proietti $(D),{ }^{1}$ Veronica Balduzzi $\left(D,{ }^{1}\right.$ \\ Nicoletta Bernardini, ${ }^{1}$ Anna Marchesiello, ${ }^{1}$ Simone Michelini, ${ }^{1}$ Ersilia Tolino, ${ }^{1}$ \\ Salvatore Volpe, ${ }^{1}$ Patrizia Maddalena, ${ }^{1}$ Antonella Ciaramella, ${ }^{2}$ Gianfranco Raimondi, \\ and Concetta Potenza ${ }_{(1 D}{ }^{1}$
}

${ }^{1}$ Department of Medical-Surgical Sciences and Biotechnologies, Dermatology Unit "Daniele Innocenzi”, Sapienza University of Rome,
Polo Pontino, Italy
${ }^{2}$ Department of Internal Medicine, Sapienza University, Rome, Italy

Correspondence should be addressed to Ilaria Proietti; proiettilaria@gmail.com

Received 4 March 2020; Revised 18 May 2020; Accepted 2 June 2020; Published 1 July 2020

Guest Editor: Annalisa Romani

Copyright (c) 2020 Nevena Skroza et al. This is an open access article distributed under the Creative Commons Attribution License, which permits unrestricted use, distribution, and reproduction in any medium, provided the original work is properly cited.

Hidradenitis suppurativa (HS) is a chronic inflammatory skin disease associated with elevated prevalence of comorbidities, especially metabolic and cardiovascular diseases. We used a tool called Heart Rate Variability (HRV) in order to assess the correlation between HS and alterations of the sympathetic-vagal equilibrium in the autonomic cardiovascular regulation system. We found increased sympathetic activity, associated with a higher risk of cardiovascular disease. HS, according to our results, is an independent cardiovascular risk factor.

\section{Introduction}

Hidradenitis suppurativa (HS) is a chronic inflammatory skin disease characterized by recurrent skin nodules, abscesses, and draining sinus tracts affecting primarily the apocrine glandrich regions of the skin, especially the axillae and groin $[1,2]$.

HS has a chronic evolution with unpredictable periodic exacerbations. The lesions show a tendency to turn into fistulous tunnels, draining externally a serum-purulent material healing with the formation of retracting scars. Prevalence is estimated from $<1 \%$ to $4 \%[3,4]$, and the incidence is 11.4 cases per 100,000 patients, which is steadily increasing. Previous studies revealed a female predominance of $3: 1$ in the general population $[5,6]$. Genetic factors play a fundamental role in the pathogenesis of HS, and one-third of patients report a positive family history of the disease. However, although the HS pathogenetic mechanism is still unknown, follicular hyperkeratinization probably characterizes the first step of the inflammatory process [7]. Histological examination revealed follicular hyperkeratosis and lymphocytic peri- follicular infiltration followed by the disruption of the follicular unit.

Furthermore, the immunohistochemistry studies revealed increased production of proinflammatory molecules, like tumor necrosis factor- (TNF-) $\alpha$, interleukin- (IL-) $1 \mathrm{~b}$, IL-12, IL-17, and IL-23.

In addition, TNF- $\alpha$ is one of the main cytokines that can be associated with the development of insulin resistance and hyperlipidemia, characteristics associated with metabolic syndrome.

Recent works suggest that HS should be viewed as a systemic inflammatory disease linked most commonly to metabolic, gastrointestinal, rheumatologic, psychiatric, and cardiovascular (CV) comorbidities.

The prevalence of the sympathetic branch of the autonomous cardiovascular system in the population reflects an increased CV risk. In this study, we used Heart Rate Variability (HRV) as a noninvasive tool to assess the sympatheticvagal equilibrium in the autonomous $\mathrm{CV}$ regulation system [8-10]. Our aim was to evaluate whether autonomic function 
is affected in young HS patients and whether to consider HS as an independent cardiovascular risk factor [11].

\section{Material and Methods}

2.1. Subjects. Subjects are those affected by moderate to severe HS who attended our Outpatient Service of Dermatology from October 2018 to April 2019.

The heart rate (HR), RR trait (in $\mathrm{ms}$ ), PQ interval (v.n.: 120-220 ms), QRS (v.n.: <100 ms), and QTc (v.n.: <430 ms) were measured.

Patients were informed not to assume any modifiers of autonomic nervous system functions such as psychoactive drugs and caffeine within 4 hours prior to the electrocardiogram (ECG). Informed consent was collected from each patient.

2.2. Assessments. We used the Cardiolab CE pocket PC ECG system in order to obtain an $8 \mathrm{~min}$ digital ECG at rest in supine position and during 8 minutes of orthostatic position obtained by the use of a passive tilt table.

With dedicated software (Cardiolab-Xai Medica), we analyzed the HRV indexes in the time and frequency domains. We will also analyze the nonlinear dynamics of HRV by Kubios software [12].

2.3. Linear Methods. We used linear methods in order to obtain a direct estimation of HRV through two types of analysis: time-domain analysis and frequency-domain analysis.

The time-domain method is based on NN intervals which are related to RR intervals in the ECG. They are analyzed to acquire variables such as SDNN (Standard Deviation of all NN intervals) and RMSSD (Root Mean Square of Successive Differences between adjacent NNs); both indexes represent parasympathetic tone.

Frequency-domain analysis, which is based on the power spectral density of the heart rate time series, highlights the issue of the underlying rhythms of the mechanisms controlling the heart rate (HR) and identified three major spectral peaks: high frequency (HF: $0.15-0.4 \mathrm{~Hz}$ ), low frequency (LF: 0.04-0.15 Hz), and very low frequency (VLF: below $0.04 \mathrm{~Hz}$ ) in the adult HR spectrum.

LF is an indicator of both sympathetic and parasympathetic activities. HF reflects parasympathetic activity. The $\mathrm{LF} / \mathrm{HF}$ ratio is an index representing overall balance between sympathetic and parasympathetic systems. Higher values reflect dominance of the sympathetic branch, while lower ones reflect the predominance of the parasympathetic action.

2.4. Nonlinear Methods. The nonlinear methods (Poincarè plots and Detrended fluctuation analysis) are not modified by environmental features and measure the complex mechanisms regulating the signal [13].

Poincarè plots is a two-dimensional vector analysis that was used to quantify the shape of the plots (Figure 1). In this quantitative method, short-term (SD1) and long-term (SD2) RR interval variability and the ellipse area of the plot are quantified separately. SD1 is an indicator of vagal modulation of the sinus node. The HR correlations were defined separately for short-term $(<11$ beats, $\alpha 1)$ and longer-term
(>11 beats, $\alpha 2$ ) RR interval data. The $\alpha 1$ index was positively correlated with LF in normalized units. The other nonlinear method is the detrended fluctuation analysis (DFA) that consists of a procedure utilized to measure the fractal scaling assets of short-term and intermediate-term variability in the RR ECG intervals. The decrease of the $\alpha 1$ index is considered a mortality prognostic factor in patients affected by severe cardiovascular conditions such as ischemic events and cardiac insufficiency.

2.5. Data Analysis. Our study results were examined through two types of software: Cardiolab, Xai Medica (used to investigate HRV linear methods), and Kubios HRV 2.0 (for the HRV nonlinear analysis examination). We expressed our data as the mean \pm standard error. We calculated the $t$-test in order to estimate the quantitative variables, and the chisquared test was performed to estimate the qualitative ones.

We performed a statistical analysis using the software SigmaStat 3.5 (Systat Software Inc., Point Richmond, CA, USA). We considered a $P$ value with a significance level $<0.05$.

\section{Results}

Sixteen HS patients were recruited, including 9 females and 7 males aged between 16 and 48 years. Four of sixteen patients have normal weight, 6/16 were overweight, 3/16 patients presented with grade I obesity, 2/16 patients presented grade II obesity, and one patient presented with grade III obesity. In addition, eight of sixteen patients were smokers.

Information about gender, age, weight, height, BMI, and smoking habits is reported in Table 1.

We did not observe any significative difference when we catalogued patients according to BMI, though an increase of the heart rate (RR) was observed in subjects with a BMI $>25$ (Table 2).

On the difficulty of having clean data, we analyzed 8/16 patients in detail, as preliminary data: 3 males and 5 females aged between 16 and 48 years. Of these subjects, only 2 patients presented with a normal weight while 6 patients had a BMI greater than $24.99 \mathrm{~kg} / \mathrm{m}^{2}$ (2 patients were overweight, 3 patients had a grade I obesity, and 1 patient had a grade III obesity). Five patients were smokers, and 3 patients were nonsmokers. In all patients, the age of onset was between 10 and 28 years while the age of diagnosis was between 15 and 45 years. The ECG recorded did not show any alteration, except in one patient, where it showed a right conduction disorder (Table 3).

As expected, from the supine to orthostatic position, RR interval showed a significant decrease (from $796.3 \pm 110.8$ to $674.68 \pm 56.95 \mathrm{msec}-P<0.001)$; systolic and diastolic arterial pressure showed a slight increase as in normal subjects (Tables 4 and 5).

We also compared the HRV indexes of subjects with HS with those observed in a group of 14 normal subjects from our previous work. We observed a reduction of total HRV in the time and frequency domain: the STD showed a significant decrease in HS patients $(40.32 \pm 5.79$ to $33.1 \pm 19.4 \mathrm{msec})$. The sympathetic indexes in HS patients are higher than those 

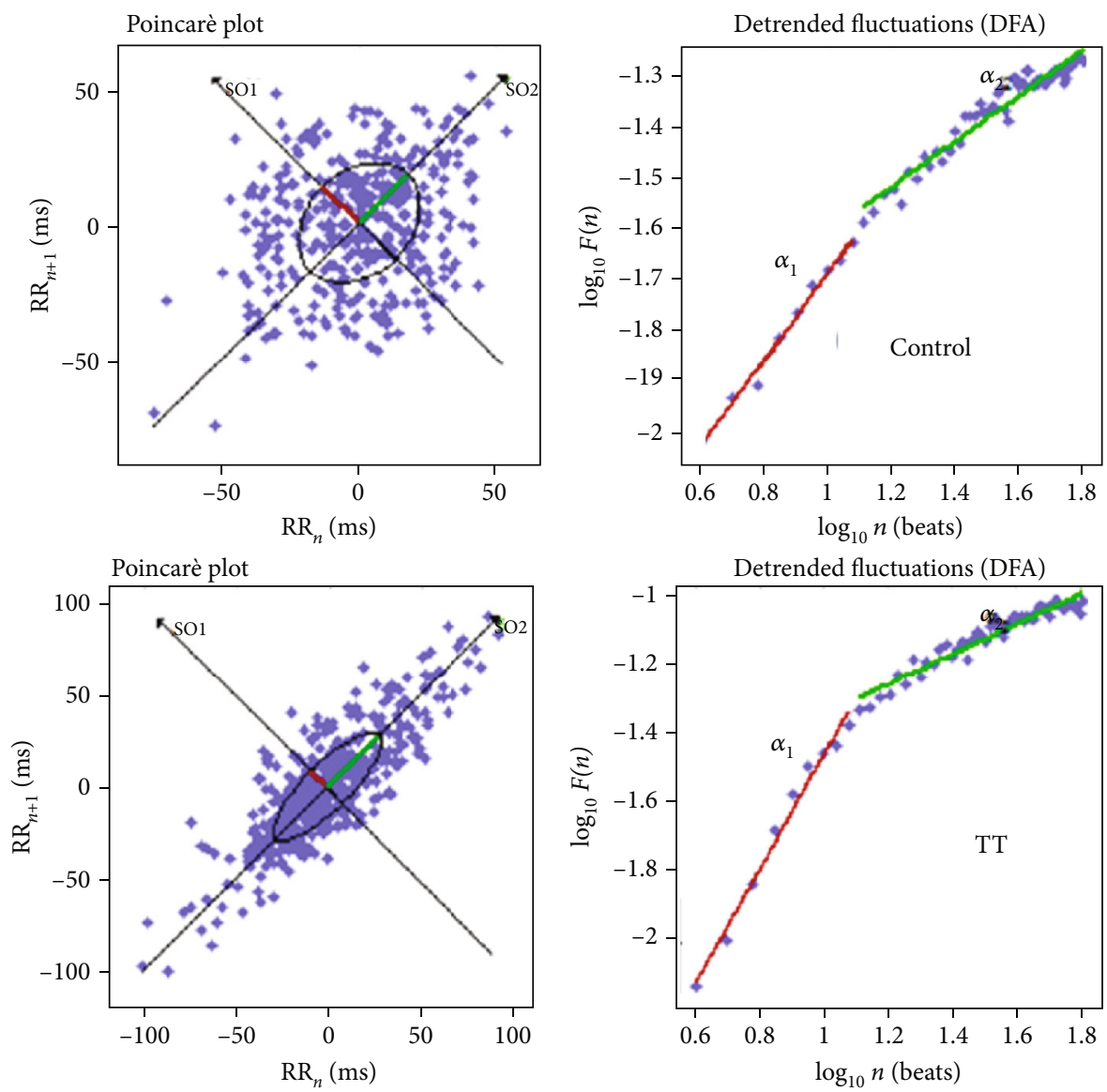

Figure 1: An example of Poincarè plot and DFA during a Tilt Test (TT) in a patient with HS.

TABLE 1: HS patients included in the study and assessed with ECG.

\begin{tabular}{lcccccccccc}
\hline Age (years) & Sex & Height $(\mathrm{cm})$ & Weight $(\mathrm{kg})$ & BMI & Smoke & HR $(\mathrm{bpm})$ & RR $(\mathrm{ms})$ & PQ $(\mathrm{ms})$ & QRS (ms) & QTc $(\mathrm{ms})$ \\
\hline 41 & F & 1.68 & 83 & 29.4 & Yes & 78 & 768 & 114 & 88 & 324.00 \\
16 & F & 1.65 & 72 & 26.4 & No & 93 & 642 & 148 & 58 & 296.00 \\
31 & M & 1.7 & 95 & 32.9 & Yes & 81 & 740 & 140 & 92 & 344.00 \\
15 & M & 1.62 & 93 & 35.4 & No & 87 & 690 & 140 & 78 & 332.00 \\
48 & M & 1.8 & 90 & 27.8 & Yes & 60 & 1004 & 170 & 84 & 410.00 \\
13 & F & 1.42 & 79 & 39.2 & No & 89 & 672 & 134 & 80 & 303.00 \\
28 & F & 1.7 & 85 & 29.4 & Yes & 74 & 816 & 98 & 70 & 361.00 \\
16 & M & 1.85 & 85 & 24.8 & No & 59 & 1020 & 152 & 88 & 404.00 \\
36 & M & 1.66 & 77 & 27.9 & No & 70 & 856 & 148 & 88 & 370.00 \\
19 & F & 1.76 & 72 & 23.2 & No & 78 & 768 & 122 & 76 & 324.00 \\
32 & F & 1.65 & 83 & 30.5 & Yes & 79 & 758 & 148 & 86 \\
28 & F & 1.57 & 76 & 30.8 & Yes & 67 & 892 & 144 & 84 & 322.00 \\
24 & F & 1.7 & 130 & 45 & Yes & 93 & 644 & 156 & 88 & 297.00 \\
16 & F & 1.67 & 60 & 21.5 & No & 83 & 720 & 172 & 108 \\
22 & M & 1.85 & 95 & 27.8 & Yes & 74 & 806 & 104 & 110 \\
21 & M & 70 & 1.85 & 20.5 & No & 69 & 866 & 120 & 88 \\
\hline 25.38 & & 6.23 & 79.80 & 29.53 & & 77.125 & 791.375 & 138.125 & 85.375 & 372.00 \\
\hline
\end{tabular}


TABLE 2: HS patients included in the study and assessed with ECG, divided by BMI.

(a) Normal weight

\begin{tabular}{lcccccc}
\hline Physical condition & Smoke & HR & RR & PQ & QRS & QTc \\
\hline Normal weight & No & 59 & 1020 & 152 & 88 & 404.00 \\
Normal weight & No & 78 & 768 & 122 & 76 & 324.00 \\
Normal weight & No & 83 & 720 & 172 & 108 & 314.00 \\
Normal weight & No & 69 & 866 & 120 & 88 & 372.00 \\
& & 72.25 & 843.5 & 141.5 & 90 & 353.50 \\
\hline
\end{tabular}

(b) Overweight and obesity

\begin{tabular}{lcccccc}
\hline Physical condition & Smoke & HR & RR & PQ & QRS & QTc \\
\hline Obesity I & Yes & 81 & 740 & 140 & 92 & 344.00 \\
Obesity I & Yes & 79 & 758 & 148 & 86 & 322.00 \\
Obesity I & Yes & 67 & 892 & 144.0 & 84 & 349.00 \\
Obesity II & No & 87 & 690 & 140 & 78 & 332.00 \\
Obesity II & No & 89 & 672 & 134 & 80 & 303.00 \\
Obesity III & Yes & 93 & 644 & 156 & 88 & 297.00 \\
Overweight & Yes & 78 & 768 & 114 & 88 & 324 \\
Overweight & No & 93.0 & 642 & 148 & 58 & 296.00 \\
Overweight & Yes & 60 & 1004 & 170 & 84 & 401.00 \\
Overweight & Yes & 74 & 816 & 98 & 70 & 361.00 \\
Overweight & No & 70 & 856 & 148 & 88 & 370.00 \\
Overweight & Yes & 74 & 806 & 104 & 110 & 359.00 \\
& & 78.75 & 774 & 137 & 85.38 & 338.17 \\
\hline
\end{tabular}

in normal subjects even if not all of them reached statistical significance (LF/HF ratio $1.13 \pm 0.24$ vs. $1.42 \pm 1.1$, DFA $\alpha 1$ $0.85 \pm 006$ vs. $1.04 \pm 0.29$ ).

\section{Discussion}

HS is an inflammatory skin disease of the terminal hair follicle. It manifests clinically with the onset of painful nodules, abscesses, and draining sinus tracts eventually resulting in scars, involving multiple regions of the body.

Some recent studies have suggested a connection between HS and a significantly increased risk of ischemic cardiovascular events, such as myocardial infarction and stroke. It was also suggested that the risk of ischemic events is higher in subjects affected by HS when compared to severe psoriasis patients $[14,15]$. The association between inflammatory markers and sympathetic hyperactivation indexes remained significant after correcting important risk factors and confounding factors such as age, Body Mass Index, heart rate, and smoking. Sympathetic activation can also be triggered by reflex mechanisms (arterial baroreceptor impairment), psychological stress, oxidative stress, obstructive sleep apnea, inflammation, and metabolic factors as I.R. and dysregulated production and secretion of adipokines from visceral fat with a particular important role of leptin [16].

Interestingly, a Danish cross-sectional study showed that the mean heart rate in severe HS patients in resting condi- tions was suggestively higher when compared with that of controls [17]. Both HS and atherosclerosis have a chronic inflammatory subset, and cardiovascular and metabolic diseases are the most common comorbidities found in HS $[18,19]$. Atherosclerosis, a chronic inflammatory disease affecting the blood vessels, is considered a major cause of cardiovascular diseases. Markers of inflammation are associated with both cardiovascular risk and the severity of HS inflammatory manifestations. It is known that inflammation represents the core in the bridge between HS and the pathogenesis of cardiovascular diseases, though many doubts remain regarding its mechanism of action. An important role is played by interleukin- (IL-) 17, which is found to be upregulated in lesional and perilesional skin of HS patients [20]. Overexpression of various other cytokines, such as IL-1, TNF- $\alpha$, IL-10, and IL-11, as well as antimicrobial peptides (AMPs), beta-defensin 2, psoriasin, and cathelicidin, has been observed in the lesional setting.

Interleukin- (IL-) 32 expression is significantly enhanced in plaque of atherosclerosis, and its proinflammatory function could help increase the risk of CV events in patients affected by chronic inflammatory diseases [21, 22]. Moreover, HS patients generally present a higher incidence of other cardiovascular risk factors such as obesity, smoking history, diabetes, hypertriglyceridemia, and metabolic syndrome [23].

The present study was based on the analysis of HRV as a noninvasive tool to evaluate the association between HS and an increased CV risk. The mechanism leading to depressed HRV in heart failure is complex and not perfectly described, but it could partially be related to the alteration of neurohumoral activity [24].

The results indicate that the sympathetic activity increases during the Tilt Test more than in normal subjects as shown by the tachycardia and by the increase of the arterial pressure and LF/HF ratio and the decrease of the STD. The Tilt Test induces, also, an increase in the properties of short-term fractal correlations of heart rate dynamics (DFA) accompanied with a decrease in all the nonlinear indexes of HRV, confirming the thesis that these indexes are an expression of the cardiac sympathetic-vagal balance.

HS patients, if compared to the rest of the population, tend to present a higher incidence of carotid atherosclerosis. The role of smoking in the severity of HS is still not clear because it is not known whether smoking cessation improves the course of the disease.

A higher resting heart rate and a lower heart rate variability are linked to subclinical chronic inflammation in adult and elderly patients. The higher death rate that was described in this kind of situations could therefore manifest a shared etiology. A disproportion of the sympathetic branch of the autonomic nervous system could correlate with inflammatory events to play a major role in atherosclerosis.

It has been previously demonstrated that the use of HRV allows us to evidence an upregulated CV risk in other inflammatory dermatological disorders, such as psoriasis [25]. As reported in patients with psoriasis, in patients with $\mathrm{HS}$, the sympathetic activation indexes (LF/HF, SD2/SD1, and $\alpha 1 / \alpha 2)$ are higher than those in normal subjects too. 
TABLE 3: Characteristics of HS patients evaluated with HRV (Heart Rate Variability).

\begin{tabular}{|c|c|c|c|c|c|c|c|c|}
\hline Age & Weight (kg) & Height (m) & BMI & Physical condition & Smoke & Age of onset & Age of diagnosis & ECG \\
\hline 16 & 72 & 1.65 & 26.4 & Overweight & No & 10 & 15 & Normal \\
\hline 31 & 95 & 1.7 & 32.9 & Obesity I & Yes & 20 & 30 & Normal \\
\hline 48 & 90 & 1.8 & 27.8 & Overweight & Yes & 20 & 45 & $\mathrm{dx}$ conduction disorder \\
\hline 19 & 72 & 1.76 & 23.2 & Normoweight & No & 14 & 18 & Normal \\
\hline 32 & 83 & 1.65 & 30.5 & Obesity I & Yes & 28 & 28 & Normal \\
\hline 28 & 76 & 1.57 & 30.8 & Obesity I & Yes & 15 & 25 & Normal \\
\hline 24 & 130 & 1.7 & 45 & Obesity III & Yes & 13 & 23 & Normal \\
\hline 21 & 70 & 1.85 & 20.5 & Normoweight & No & 14 & 20 & Normal \\
\hline
\end{tabular}

TABLE 4: HRV values obtained using both linear and nonlinear methods in clinostatic position.

\begin{tabular}{ccccccccccccc}
\hline Clino & RR (ms) & STD RR (ms) & LF (\%) & HF (\%) & LF/HF (-) & SD $_{1}(\mathrm{~ms})$ & $\mathrm{SD}_{2}(\mathrm{~ms})$ & $\mathrm{SD}_{2} / \mathrm{SD}_{1}$ & $\alpha_{1}$ & $\alpha_{2}$ & $\alpha_{1} / \alpha_{2}$ \\
\hline & 675 & 24.5 & 71 & 23.2 & 3.06 & 12.6 & 32.3 & 0.39 & 1.411 & 0.302 & 4.67 \\
& 757.1 & 16.9 & 67.3 & 23.8 & 2.83 & 11.5 & 20.9 & 0.55 & 1.272 & 0.46 & 2.77 \\
& 965.5 & 34.3 & 33.3 & 62.1 & 0.54 & 33.3 & 35.4 & 0.94 & 0.627 & 0.372 & 1.69 \\
& 795.8 & 36.6 & 40.9 & 51.5 & 0.79 & 27.9 & 43.7 & 0.64 & 0.839 & 0.436 & 1.92 \\
& 766.2 & 26.1 & 38.8 & 49.7 & 0.78 & 19.1 & 31.7 & 0.6 & 1.015 & 0.443 & 2.29 \\
& 860.6 & 42.3 & 29.6 & 62.3 & 0.48 & 19.6 & 28.5 & 0.69 & 0.69 & 0.39 & 1.77 \\
& 642.8 & 10.4 & 55.6 & 31 & 1.79 & 7 & 12.9 & 0.54 & 1.024 & 0.525 & 1.95 \\
& 907.6 & 73.6 & 35.9 & 61.4 & 0.58 & 57.1 & 87.1 & 0.66 & 0.829 & 0.203 & 4.08 \\
\hline Mean & 796.3 & 33.1 & 46.6 & 45.6 & 1.4 & 23.5 & 36.6 & 0.6 & 1 & 0.4 & 2.6 \\
SD & 110.8 & 19.4 & 15.9 & 17.1 & 1.1 & 16.1 & 22.4 & 0.2 & 0.3 & 0.1 & 1.1 \\
\hline
\end{tabular}

TABLE 5: HRV values obtained using both linear and nonlinear methods in orthostatic position.

\begin{tabular}{lcccccccccccc}
\hline Ortho & RR (ms) & STD RR (ms) & LF (\%) & HF (\%) & LF/HF (-) & SD $_{1}(\mathrm{~ms})$ & $\mathrm{SD}_{2}(\mathrm{~ms})$ & $\mathrm{SD}_{1} / \mathrm{SD}_{2}$ & $\alpha_{1}$ & $\alpha_{2}$ & $\alpha_{1} / \alpha_{2}$ \\
\hline & 599.5 & 27.9 & 79.1 & 7.5 & 10.55 & 9 & 38.4 & 0.23 & 1.714 & 0.486 & 3.53 \\
& 724.5 & 18.5 & 63.2 & 23.4 & 2.70 & 9.6 & 24.4 & 0.39 & 1.401 & 0.558 & 2.51 \\
& 746.7 & 30.3 & 57.6 & 34.4 & 1.67 & 25.8 & 34.2 & 0.75 & 0.975 & 0.373 & 2.61 \\
& 741.8 & 25.3 & 47.7 & 46.9 & 1.02 & 14.3 & 32.8 & 0.44 & 1.126 & 0.422 & 2.67 \\
& 669.8 & 20.2 & 63.9 & 20.7 & 3.09 & 11 & 26.3 & 0.42 & 1.299 & 0.63 & 2.06 \\
& 646.8 & 23.2 & 86 & 5.3 & 16.23 & 6.8 & 32.1 & 0.21 & 1.759 & 0.522 & 3.37 \\
& 614.3 & 13.7 & 54.6 & 36 & 1.52 & 7.6 & 17.8 & 0.43 & 1.080 & 0.576 & 1.88 \\
& 654 & 41.8 & 54.4 & 39.3 & 1.38 & 23.3 & 54.4 & 0.43 & 1.198 & 0.329 & 3.64 \\
\hline \multirow{2}{*}{ Mean } & 674.68 & 25.11 & 63.31 & 26.69 & 4.77 & 13.43 & 32.55 & 0.41 & 1.32 & 0.49 & 2.78 \\
SD & 56.95 & 8.58 & 13.07 & 15.05 & 5.57 & 7.26 & 10.94 & 0.16 & 0.29 & 0.10 & 0.66 \\
\hline
\end{tabular}

Studies conducted on patients with known HS indicate that many of them are overweight. Obesity is one of the main risk factors associated with the development of HS, although its role is yet to be defined. We did not observe any significant difference when reorganizing patients according to the BMI, although an increase of heart rate (RR) was observed in subjects with a Body Mass Index $(\mathrm{BMI})>25$. Furthermore, we observed that the sympathetic activation indexes during passive orthostatism had increased compared to clinostatism.

\section{Conclusions}

Our data show a balanced reduction of the parasympathetic influence on the sinus node in patients with moderate to severe HS. These results could correlate with an increased risk of cardiovascular disease; thus, HS should be considered as an independent $\mathrm{CV}$ risk factor.

Our data seems to indicate an increase in the sympathetic hyperactivity indices in HS patients, but since our study was based on a limited number of cases, we consider it to be 
preliminary and more studies are necessary in order to obtain statistical significance. Identification of individuals at risk for subsequent morbidity and nonrisk groups requires future prospective studies to determine the sensitivity, specificity, and predictive values of HRV.

Moreover, our data demonstrates that biological therapy and other anti-inflammatory therapies could alter the autonomous cardiac function in patients with moderate to severe HS, as reported in a previous work on psoriasis [26]. The important connection between HS and cardiovascular diseases underlines the necessity of a cardiovascular screening in HS patients, particularly if other risk factors are present.

Although the exact role of smoking in the pathogenesis of HS remains to be determined, HS could give us another opportunity to encourage our patients to change their lifestyle.

Dermatologists must be conscious of the comorbidities related to HS. The aim is to create a condition in which patients are managed by the proper specialists. This may lead to an improvement in the disease course and in the quality of life of patients affected by this disease.

\section{Data Availability}

The authors are available to provide data supporting the research.

\section{Conflicts of Interest}

The authors declare that there is no conflict of interest regarding the publication of this article.

\section{Acknowledgments}

The authors thank Maris Garori for her writing assistance, technical editing, and language editing.

\section{References}

[1] G. B. E. Jemec and A. B. Kimball, "Hidradenitis suppurativa: Epidemiology and scope of the problem," Journal of the American Academy of Dermatology, vol. 73, no. 5, pp. S4-S7, 2015.

[2] C. Vinkel and S. F. Thomsen, "Hidradenitis Suppurativa: Causes, Features, and Current Treatments," The Journal Of Clinical And Aesthetic Dermatology, vol. 11, no. 10, pp. 1723, 2018.

[3] A. Alikhan, P. J. Lynch, and D. B. Eisen, "Hidradenitis suppurativa: A comprehensive review," Journal of the American Academy of Dermatology, vol. 60, no. 4, pp. 539-561, 2009.

[4] I. Cosmatos, A. Matcho, R. Weinstein, M. O. Montgomery, and P. Stang, "Analysis of patient claims data to determine the prevalence of hidradenitis suppurativa in the United States," Journal of the American Academy of Dermatology, vol. 68, no. 3, pp. 412-419, 2013.

[5] A. Garg, J. S. Kirby, J. Lavian, G. Lin, and A. Strunk, "Sex- and Age-Adjusted Population Analysis of Prevalence Estimates for Hidradenitis Suppurativa in the United States," JAMA dermatology, vol. 153, no. 8, pp. 760-764, 2017.

[6] J. R. Ingram, S. Hadjieconomou, and V. Piguet, "Development of core outcome sets in hidradenitis suppurativa: systematic review of outcome measure instruments to inform the process," British Journal of Dermatology, vol. 175, no. 2, pp. 263-272, 2016.

[7] K. McMillan, "Hidradenitis Suppurativa: Number of Diagnosed Patients, Demographic Characteristics, and Treatment Patterns in the United States," American Journal Of Epidemiology, vol. 179, no. 12, pp. 1477-1483, 2014.

[8] S. Akselrod, D. Gordon, F. Ubel, D. Shannon, A. Berger, and R. Cohen, "Power spectrum analysis of heart rate fluctuation: a quantitative probe of beat-to-beat cardiovascular control," Science, vol. 213, no. 4504, pp. 220-222, 1981.

[9] A. Malliani, M. Pagani, F. Lombardi, and S. Cerutti, "Cardiovascular neural regulation explored in the frequency domain," Circulation, vol. 84, no. 2, pp. 482-492, 1991.

[10] R. Balocchi, F. Cantini, M. Varanini, G. Raimondi, J. M. Legramante, and A. Macerata, "Revisiting the potential of time-domain indexes in short-term HRV analysis," Biomedizinische Technik/Biomedical Engineering, vol. 51, no. 4, pp. 190 193, 2006

[11] J. M. Legramante, S. Sacco, G. Raimondi et al., "Investigating feedforward neural regulation of circulation from analysis of spontaneous arterial pressure and heart rate fluctuations in conscious rats," American Journal of Physiology-Heart and Circulatory Physiology, vol. 296, no. 1, pp. H202-H210, 2009.

[12] G. Raimondi, J. M. Legramante, B. Scordamaglia et al., "Linear and non-linear RR interval variability analysis in the neurovegetative cardiovascular assessment in Psoriasis and Obesity," Applications of Information Systems in Engineering and Bioscience, pp. 61-69, 2014.

[13] B. Scordamaglia, I. Masci, F. Sindona, R. Cuozzo, A. Ciaramella, and G. Raimondi, "Non-linear analysis of the heart rate variability during passive Tilt test," Internal and Emergency Medicine, vol. 10, 2016.

[14] A. Egeberg, G. H. Gislason, and P. R. Hansen, "Risk of major adverse cardiovascular events and all-cause mortality in patients with hidradenitis suppurativa," JAMA Dermatology, vol. 152, no. 4, pp. 429-434, 2016.

[15] C.-T. Hung, C.-P. Chiang, C.-H. Chung, C.-H. Tsao, W.-C. Chien, and W.-M. Wang, "Increased risk of cardiovascular comorbidities in hidradenitis suppurativa: A nationwide, population-based, cohort study in Taiwan," The Journal of dermatology, vol. 46, no. 10, pp. 867-873, 2019.

[16] M. P. Canale, S. Manca di Villahermosa, G. Martino et al., "Obesity-Related Metabolic Syndrome: Mechanisms of Sympathetic Overactivity," International Journal of Endocrinology, vol. 2013, Article ID 865965, 12 pages, 2013.

[17] C. R. Juhl, I. M. Miller, G. B. Jemec, J. K. Kanters, and C. Ellervik, "Hidradenitis suppurativa and electrocardiographic changes: a cross-sectional population study," British Journal of Dermatology, vol. 178, no. 1, pp. 222-228, 2018.

[18] A. Cartron and M. S. Driscoll, "Comorbidities of hidradenitis suppurativa: A review of the literature," International Journal of Women's Dermatology, vol. 5, no. 5, pp. 330334, 2019.

[19] G. Shalom, T. Freud, I. Harman-Boehm, I. Polishchuk, and A. D. Cohen, "Hidradenitis suppurativa and metabolic syndrome: a comparative cross-sectional study of 3207 patients," British Journal of Dermatology, vol. 173, no. 2, pp. 464-470, 2015.

[20] N. Bernardini, N. Skroza, E. Tolino et al., "IL-17 and its role in inflammatory, autoimmune, and oncological skin diseases: 
state of art," International Journal Of Dermatology, vol. 59, no. 4, pp. 406-411, 2020.

[21] R. Thomi, D. Yerly, N. Yawalkar, D. Simon, C. Schlapbach, and R. E. Hunger, "Interleukin-32 is highly expressed in lesions of hidradenitis suppurativa," British Journal Of Dermatology, vol. 177, no. 5, pp. 1358-1366, 2017.

[22] V. De Vita and P. R. Hansen, "Interleukin-32 may contribute to increased risk of cardiovascular disease in patients with hidradenitis suppurativa," Atherosclerosis, vol. 267, p. 175, 2017.

[23] T. Tzellos, C. C. Zouboulis, W. Gulliver, A. D. Cohen, P. Wolkenstein, and G. B. E. Jemec, "Cardiovascular disease risk factors in patients with hidradenitis suppurativa: a systematic review and meta-analysis of observational studies," British Journal of Dermatology, vol. 173, no. 5, pp. 1142-1155, 2015.

[24] D. Aronson, M. A. Mittleman, and A. J. Burger, "Interleukin-6 Levels are Inversely Correlated with Heart Rate Variability in Patients with Decompensated Heart Failure," Journal of Cardiovascular Electrophysiology, vol. 12, no. 3, pp. 294-300, 2001.

[25] I. Proietti, G. Raimondi, N. Skroza et al., "Cardiovascular Risk in Psoriatic Patients Detected by Heart Rate Variability (HRV) Analysis," Drug Development Research, vol. 75, pp. S81-S84, 2014.

[26] C. Potenza, G. Raimondi, R. Pampena et al., "Cardiovascular risk evaluation through heart rate variability analysis in psoriatic patients before and after 24 weeks of etanercept therapy: Prospective study," Journal of International Medical Research, vol. 44, 1_suppl, pp. 43-47, 2016. 